\title{
REGISTRO ESPAÑOL DEL SISTEMA TRT(*) REMEEX EN MUJERES CON INCONTINENCIA URINARIA DE ESFUERZO.
}

\author{
Jesús Moreno Sierra?, Antonio Marqués Queimadelos², Pedro Araño Beltran³, Pedro De La \\ Fuente Pérez ${ }^{4}$, Juan Fernando Cerezuela Requena ${ }^{5}$, Eduardo Cortés Otero ${ }^{6}$, Lluis Amat Tardiu', \\ Alejandro Sousa Escandón8, Jorge Ruiz Caballero", Juan Gambini Ricapa ${ }^{10}$, Santiago Urgell \\ Morera $^{11}$, Javier Santisteban ${ }^{12}$,Magi Valls Porcel13, Feliz Arnaiz Esteban ${ }^{14}$, José Manuel \\ Camporro Fernandez ${ }^{15}$ y Angel Silmi Moyano ${ }^{16}$.
}

Hospital Clinico Universitario' (Madrid). Hospital Clinico Universitario² (Santiago de Compostela). Fundació Puigvert ${ }^{3}$ (Barcelona). Hospital 12 de Octubre 4 (Madrid). Hospital Materno Infantil (Las Palmas). Hospital Valle Hebrón 6 (Barcelona). Hospital Sant Joan de Deu (Barcelona). Hospital Comarcal Monforte de Lemos ${ }^{8}$ (Lugo). Instituto Universitario Dexeus9. (Barcelona). Hospital General de Valencia ${ }^{10}$ (Valencia). Hospital Sagrat Cor ${ }^{11}$ (Barcelona). Hospital Universitario La Paz ${ }^{12}$ (Madrid). Hospital Bellvitge ${ }^{13}$ (Barcelona). Hospital Sta Bárbara ${ }^{14}$ (Soria). Hospital De Cabueñe ${ }^{15}$ (Gijón). Hospital Clínico San Carlos ${ }^{16}$ (Madrid). España.

Resumen.- OBJETIVO: En ausencia de estudios randomizados, el Grupo de Trabajo Español Remeex inició un registro para analizar y revisar resultados en un gran grupo de pacientes con seguimiento a medio y largo plazo, para obtener conclusiones basadas en experiencias clínicas.

MÉTODOS: Se analizan prospectivamente los datos procedentes del registro de 715 pacientes que fueron operadas con una prótesis de incontinencia reajustable Remeex TRT (Tensión free Readjustable Tape) en 15 hospitales españoles, servicios de Urología 40\% y Ginecología 60\%. El registro se realizó para evaluar la seguridad y eficacia del concepto de ajustabilidad del sling para el tratamiento quirúrgico de la Incontinencia Urinaria de Esfuerzo Femenina. El grupo de trabajo español pudo evaluar los resultados del Sistema Remeex en 683 pacientes de un grupo total de 715. La edad media fue de 59.9 (rango 2 1-87) con un periodo de seguimiento medio de 23 meses (6-93). El grupo incluye: 30.2\% de

Jesús Moreno Sierra Servicio de Urología Hospital Clínico Universitario San Carlos Martín Lago, s/n 28040 Madrid. (España)

Trabajo recibido: 29 de diciembre 2005 pacientes con Incontinencia Mixta, 33. 1\% de pacientes con Déficit Esfinteriano Intrínseco urodinámico, 35.7\% de pacientes sometidas a intervenciones previas de Incontinencia, y el $54.3 \%$ a cirugías asociadas del suelo pélvico. Todas las pacientes fueron analizadas pre-operatoriamente mediante historia clínica, examen físico y cuestionario de incontiencia. En cada seguimiento, las pacientes rellenaron un cuestionario de incontinencia, se sometieron a examen físico y test de esfuerzo.

RESULTADOS: El índice de curación valorado en la última revisión fue del 92.2\%, 6.9\% de los pacientes mejoraron y el $0.9 \%$ de las pacientes fueron consideradas fracasos. La capacidad de reajuste fue utilizada en 416 casos (60.9\%) de las pacientes como una segunda fase de la cirugía durante las siguientes 24-48 horas, antes del alta hospitalaria. 80 pacientes (1 1.7\%) fueron reajustadas a medio o largo plazo lentre 6 y 8 meses después de la cirugía). En tres pacientes el nivel de soporte del cabestrillo fue reducido con éxito entre 6 y 14 meses después de la cirugía. Ninguna otra paciente sufrió dificultades de vaciado a largo plazo. Como complicaciones, al $1.7 \%$ de las pacientes se les extrajo el varitensor, debido a la persistencia de seroma abdominal. Un $0.8 \%$ presentaron exposición del cabestrillo en la pared vaginal.

CONCLUSIONES: El sistema Remeex es una técnica mínimamente invasiva con resultados consistentes y mejorados incluso en grupos de pacientes no homogéneos, en los que se incluyen pacientes con déficit esfinteriano intrínseco, pacientes reintervenidas, incontinencia mixta y patología asociada. 
Palabras clave: Remeex. Incontinencia de Esfuerzo. Registro.

Summary.- OBJECTIVES: Due to the absence of randomized studies, the Spanish Reemex Working Group started a registry to analyze and review the results of a large group of patients with mid-term and long-term follow-up to obtain conclusions based on clinical experience.

METHODS: A registry of 715 patients who underwent surgical intervention for insertion of the SUI readjustable prosthesis Reemex TRT (tension free readjustable tape) in 15 Spanish hospitals 140\% urology departments, $60 \%$ gynecology departments). The registry was established to evaluate the safery and efficacy of the sling adjustability concept in the surgical treatment of female SUl. The Spanish Working Group was able to evaluate results of the Reemex system in 683 patients of a total of 715. Mean age was $59.9 \mathrm{yr}$. (range 21-87) with a mean follow-up of 23 months (6-93). The group includes: $30.2 \%$ patients with mixed incontinence, $73.7 \%$ patients with urodynamic intrinsic sphincteric deficit, $35.7 \%$ patients with previous history of failed surgical interventions for urinary incontinence, and $54.3 \%$ previous pelvic floor associated operations. All patients were evaluated preoperatively with history, physical examination and urinary incontinence questionnaire. Each follow-up visit included incontinence questionnaire, physical examination and stress test.

RESULTS: Cure rate was $92.2 \%$, with $6.9 \%$ improvement and $0.9 \%$ failures. Readjustment was performed in 416 patients (60.9\%) as a second phase of surgery over the following 24-48 hours, before hospital discharge. 80 patients $(11.7 \%$ ) were readjusted in the mid- or long-term (between 6 and 8 months after surgery). The level of support of the sling was successfully reduced in three patients between 6 and 14 months after surgery. No other patient suffered voiding difficulties in the long-term. $1.7 \%$ of the patients needed extraction of the tensor due to persistent abdominal wall seroma. $0.8 \%$ presented vaginal extrusion of the sling.

CONCLUSIONS: The Reemex system is a minimally invasive technique with consistent results and even improved in heterogeneous groups of patients including intrinsic sphincter defficiency, reoperations, mixed incontinence and associated pathologies.

Keywords: Remeex. Stress urinary incontinence. Registry.

\section{INTRODUCCIÓN}

Generalmente se considera que la Incontinencia Urinaria de Esfuerzo es signo o síntoma causado por la disminución de soporte del suelo pélvico sobre la uretra. Cuando a una paciente se le indica una intervención quirúrgica por su Incontinencia Urinaria de Esfuerzo, el objetivo de dicha intervención será recuperar el nivel de soporte original del suelo pélvico para este paciente en concreto. Es obvio que cada paciente puede tener un nivel diferente de afectación patológica de suelo pélvico, diferente actividad física, diferente Índice de Masa Corporal, diferente historia clínica y etiología de su incontinencia de esfuerzo.

Muchos estudios han analizado las técnicas disponibles para solventar la incontinencia de esfuerzo femenina. Así, la técnica de Burch está siendo sustituida por las cirugías con mallas libres de tensión. Sea como fuere un alto porcentaje de pacientes intervenidas con cualquier ténica antiincontinencia recidivaran a medio o largo plazo. En este sentido, el sistema Remeex se coloca sin tensión y se ajusta, si es necesario, el día después de la cirugía con la paciente de pie y realizando los esfuerzos que le provocan la incontinencia o incluso meses o años después de su implante.

El principal argumento para empezar a utilizar el dispositivo Remeex fue la posibilidad de adaptar clínicamente el nivel de soporte de la malla a cada paciente, no sólo después de la intervención, sino también a medio y largo plazo después de la misma, en caso de requerirlo, ofreciendo la posibilidad de recuperar la continencia a largo plazo, sin tener que someterse a reintervenciones futuras. En este trabajo analizados los resultados obtenidos del registro español, con 683 pacientes complicadas y también analizamos los resultados en diferentes subgrupos de pacientes.

\section{MATERIAL Y MÉTODOS}

Hemos realizado un estudio prospectivo que incluyó a 715 pacientes a las que se les indicó una intervención para tratar su incontinencia urinaria de esfuerzo con Sistema Remeex en 15 hospitales diferentes entre marzo de 1.996 y diciembre de 2.003. Trece hospitales indican el Sistema Remeex como una técnica para pacientes complicadas (reintervenciones, DEl, patología asociada), usando técnicas con mallas libres de tensión para primeras intervenciones con uretra hipermóvil. En dos de los hospitales de utiliza Remeex en todo tipo de pacientes. 
La edad media de las pacientes fué de 59.1 años, con un rango entre 21 y 87.206 pacientes $(30.2 \%)$ tenían incontinencia mixta clínica, $226(33.1 \%)$ DEl que se definió como VLPP $<60 \mathrm{~cm} \mathrm{H} 2 \mathrm{O}$ y/o PUMC $<20 \mathrm{~cm} \mathrm{H} 2 \mathrm{O} .244$ pacientes (35.7\%) se habían sometido a cirugías previas de incontinencia, con una media de cirugías previas de 1,35 (rango 1-4).

En 371 pacientes $(54.3 \%)$ se les realizó una cirugía asociada de suelo pélvico en el mismo acto quirúrgico, 235 (34.4\%) cistocele, 200 (29.3\%) rectocele, y $65(9.5 \%)$ enterocele.

El índice de curación se definió como test de esfuerzo negativo con vejiga llena (250-300 ml), sin necesidad de compresas. En este estudio se definió la mejoría como una reducción del $75 \%$ en el número de compresas por día.

A todas las pacientes se las sometió a estudio urodinámico pre-operatorio, un test de esfuerzo (por tos) en posición tumbada y de pie con un volumen en vejiga de aproximadamente $300 \mathrm{ml}$, diario miccional y medidas de volúmenes de orina residuales. En cada seguimiento, las pacientes rellenaban un cuestionario de incontinencia de calidad de vida (QoL) tal y como describimos previamente $[1,2]$, examen físico $y$ test de esfuerzo.

También se realiza examen ginecológico y análisis de orina. Después de la intervención las medidas se repiten a los 6 meses, y cada 12 meses. La media del último seguimiento es de 23 meses, y el seguimiento máximo 96 meses ( 8 años).

\section{INTERVENCIÓN}

El Sistema Remeex se colocó siguiendo la técnica quirúrgica descrita [3]. (Figura 1).

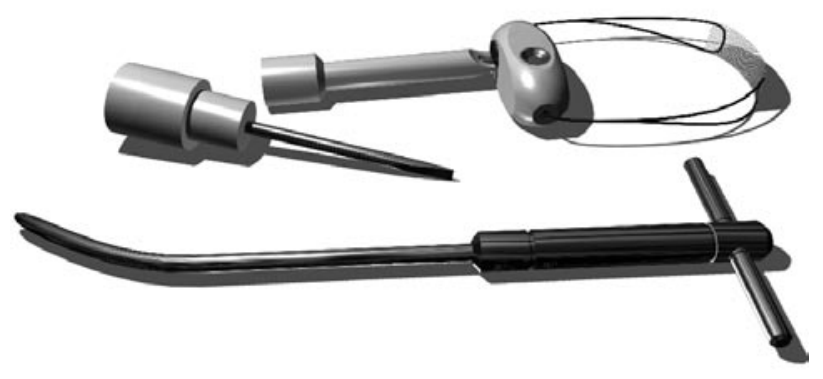

FIGURA 1. Sistema Remeex.
El Sistema Remeex puede implantarse con anestesia regional y en algunos pacientes con anestesia general. El procedimiento quirúrgico consistió en una pequeña incisión $(3 \mathrm{~cm})$ por encima del hueso púbico y disección de la grasa hasta que la facia de los rectos queda expuesta. Después de eso se realizó una incisión vaginal y se pasaron las agujas a través del espacio de Retzius, se realizó una cistoscopia para asegurar la integridad de la vejiga y se colocó la malla. Los hilos de polipropileno se introdujeron en los agujeros de las agujas pasa-hilos para llevarlos a la zona abdominal a través del espacio de Retzius. En este momento los hilos se introdujeron en el varitensor (un dispositivo de forma ovalada fabricado de polietileno biocompatible de alta densidad (chirulen) y titanio). Los hilos de tracción se ataron entre sí manteniendo el varitensor aproximadamente a $10 \mathrm{cms}$ por encima de la fascia de los rectos $y$, rotando el manipulador en el sentido de las agujas del reloj, los hilos empezaban a enrollarse dentro del varitensor. Se rotó el manipulador hasta que el varitensor estaba $3 \mathrm{cms}$ por encima de la fascia de los rectos. Entonces se cerraba la incisión abdominal dejando el manipulador asomando por fuera de la piel. Se colocó un cateter rígido en la uretra y se movió arriba y abajo para asegurar que el sling no estaba ejerciendo tensión sobre la uretra.

A la mañana siguiente a la intervención (el reajuste se hizo entre 1 y 4 días después de la intervención) se realizó el primer ajuste del nivel de soporte del sling con la paciente de pie, realizando maniobras de Valsalva con un volumen en vejiga de 250-300 ml. Si la paciente presentaba incontinencia, el manipulador se rotaba 4 giros completos en sentido horario y se repetía el test de esfuerzo. Esta

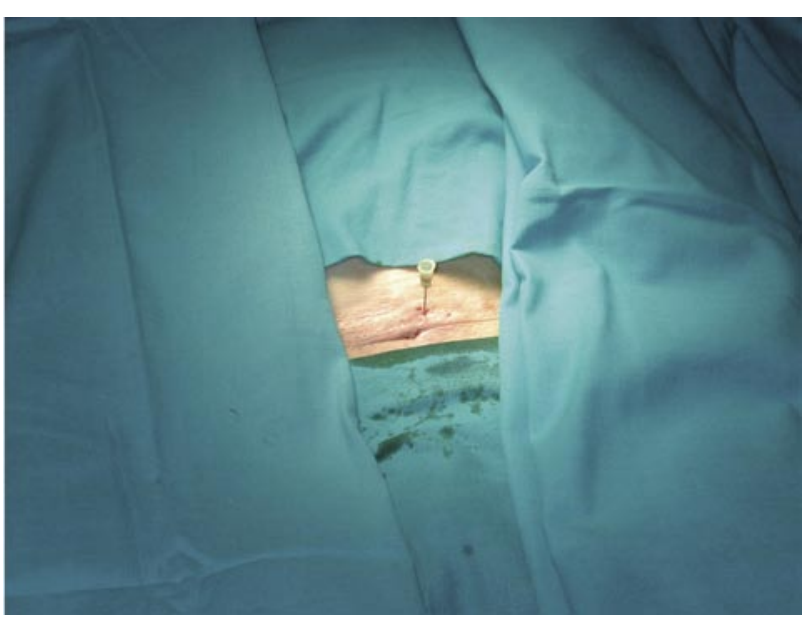

FIGURA 2. Se realiza una pequeña incisión y se inyecta anestesia local. 
maniobra se repitió hasta que la paciente alcanzaba la continencia. Acto seguido, se le pedía que orinase y se medía el residuo de orina en vejiga. Si la orina residual estaba por encima de $100 \mathrm{ml}$, se disminuía el nivel de soporte del sling. Antes del alta de la paciente el manipulador se desconectaba del varitensor utilizando el desconector.

El reajuste a medio-largo plazo se realizó cada vez que se necesitó mediante una pequeña manipulación de 10 a 20 minutos de duración. Inyectando anestesia local en la incisión abdominal, se realizó una pequeña incisión (Figura 2) y se localizó el varitensor en su cápsula de tejido fibrótico. Se abrió la cápsula (Figura 3), el manipulador se conectó al varitensor y se cerró la incisión (Figura 4). A continuación se procede al llenado de la vejiga con $300 \mathrm{ml}$ de suero salino a temperatura corporal, y con la paciente en bipedestación, se pidió a la paciente que realizara maniobras de Valsalva, reajustando el nivel de soporte del sling hasta alcanzar el nivel adecuado de continencia. Tras realizar el reajuste, se desconectó el manipulador del varitensor utilizando el desconector.

En los casos en que se precisa extraer o retirar el varitensor, se inyecta anestesia local en la incisión abdominal, localizando el varitensor, se mantiene el nivel de tensión de los hilos utilizando dos clamps protegidos con tubo de silicona, se extrajo el hilo del varitensor y se sacó el varitensor. (Figuras 2, 3 y 4$)$.

El nivel de soporte correcto de la malla se preservó anudando los extremos de los hilos de trac- ción al nivel del clampaje de los hilos. El sistema quedó convertido en ese momento en una malla bien ajustada pero sin posibilidad de reajuste posterior.

\section{RESULTADOS}

El índice de curación tras el último seguimiento fue del $92.2 \%$, con un $6.9 \%$ adicional de pacientes mejoradas. El $0.9 \%$ de las pacientes fueron consideradas fracasos. La ajustabilidad fue utilizada en 416 de las pacientes $(60.9 \%)$ como una extensión de la intervención durante las siguientes 24 - 48 horas, antes del alta hospitalaria. 80 pacientes (11.7\%) fueron reajustadas a medio-largo plazo (6-18 meses después de la cirugía).

No se observaron complicaciones mayores durante las cirugías, a parte de $7.9 \%$ de perforaciones vesicales (un alto porcentaje de las cuales fueron en pacientes recurrentes), y $3.2 \%$ de hematoma retropúbico sin consecuencias mayores. 416 pacientes $(60.9 \%)$ fueron reajustadas tras la intervención hasta llegar al punto de continencia, que fue conseguido en 671 pacientes $(98.2 \%)$ antes del alta hospitalaria. La estancia media para el reajuste fue de 2.3 días (0-14). La hospitalización media total fue de 5.6 días, debido a la gran cantidad de intervenciones de suelo pélvico asociadas. $6.4 \%$ de las pacientes tuvieron problemas de vaciado iniciales entre 2 y 4 días, que desaparecieron espontáneamente. En tres pacientes con componente obstructivo demostrado urodinamicamente el nivel de soporte de la malla fue reducido con éxito entre 6 y 12 meses tras la cirugía. En dos pacientes la malla fue destensada con éxito

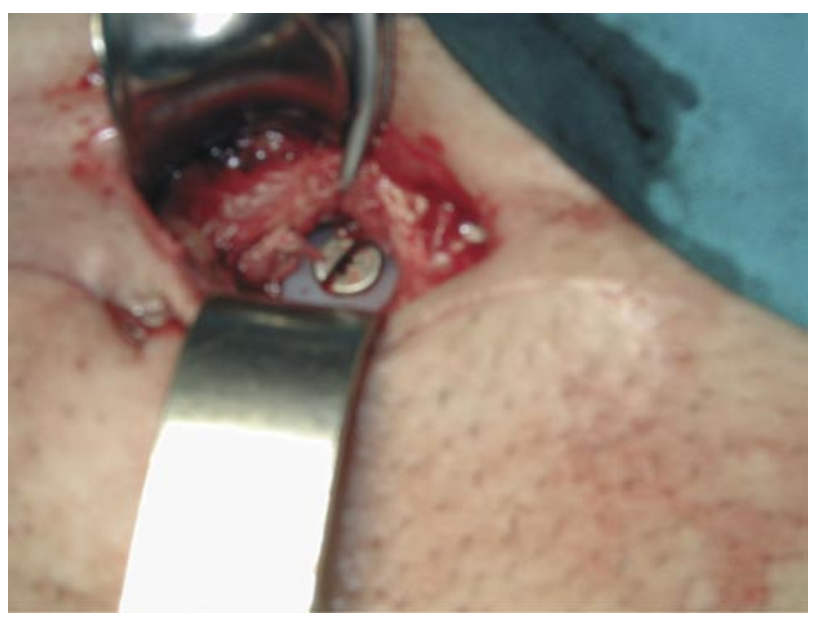

FIGURA 3. Se localiza el varitensor en su cápsula de tejido fibrótico y se abre la cápsula.

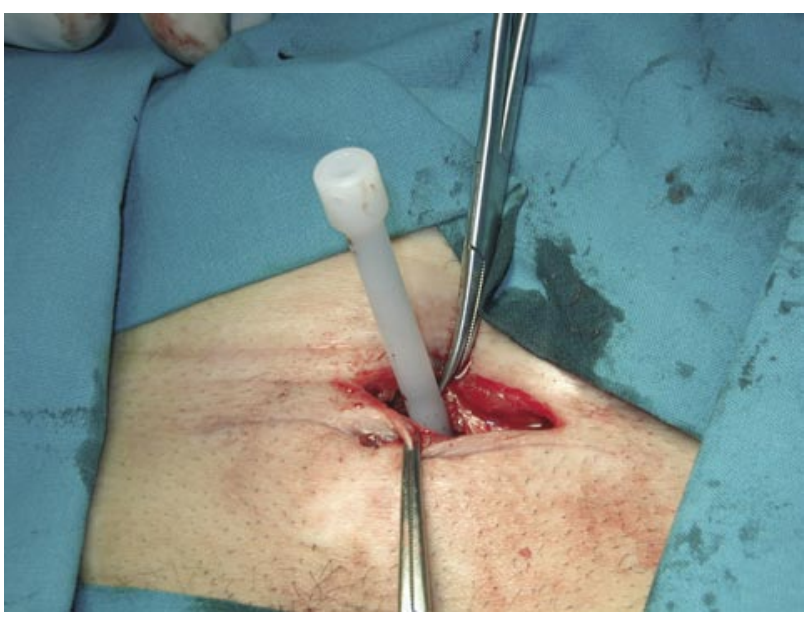

FIGURA 4. El manipulador se conecta al varitensor y se cierra la incisión. 


\section{TABLA I. SEGUIMIENTO DE LOS PACIENTES.}

\begin{tabular}{|lll|}
\hline & Seguimiento> 24 Meses & Seguimiento > 42 Meses \\
\hline Pacientes & 272 & 62 \\
\hline Seguimiento Meses / Medio & $24-93 / 36.3$ & $42-93 / 55.9$ \\
\hline Curación \# / \% & $253 / 93 \%$ & $59 / 95.2 \%$ \\
\hline Mejoria \# /\% & $14 / 5.1 \%$ & $2 / 3.2 \%$ \\
\hline Fracaso\# / \% ${ }^{*}$ ) & $5 / 1.8 \%$ & $1 / 1.6 \%$ \\
\hline Urgencia De Novo \# /\% & $17 / 6.3 \%$ & $3 / 4.8 \%$ \\
\hline Reajuste Medio-Largo plazo \# /\% & $44 / 16.2 \%$ & $8 / 12.9 \%$ \\
\hline Retirada del Varitensor \# / \% & $8 / 2.9 \%$ & $1 / 1.6 \%$ \\
\hline Erosión vaginal/\# / \% & $3 / 1.1 \%$ & $0 / 0 \%$ \\
\hline
\end{tabular}

$\left({ }^{\star}\right)$ Dos de estos cinco pacientes están pendientes de reajustar en la actualidad.

para eliminar una inestabilidad de novo. Esta capacidad de un sistema reajustable para eliminar algunas inestabilidades de novo es un aspecto que tiene que ser estudiado en más pacientes. En 52 pacientes 17.6\% apareció inestabilidad de novo, la mayoría de ellas tratadas con éxito mediante medicación anticolinérgica. Es remarcable que de 206 pacientes con síntomas de urgencia previos, 159 (77\%) se vieron aliviados post-operatoriamente.

Durante el primer seguimiento a 6 meses, 32 pacientes fueron reajustadas para mejorar su continencia. Todos los reajustes fueron satisfactorios y se realizaron mediante un procedimiento sencillo de entre 10 y 20 minutos. En esta fase de seguimiento el varitensor fue retirado en un $1,7 \%$ de las pacientes debido a seroma persistente y el $0.8 \%$ de las pacientes tenían una exposición de la malla en vagina. Esto fue reparado suturando de nuevo la incisión vaginal y extrayendo el sling en tres casos $(0.4 \%)$.

En la Tabla I analizamos dos grupos de pacientes, aquellos con más de 24 meses de seguimiento (272) y un grupo con pacientes con más de 42 meses de seguimiento (62 pacientes).

En la Tabla II podemos ver los resultados de dos subgrupos de pacientes: el subgrupo de DEl y el subgrupo de recurrentes, con un seguimiento mínimo de 24 meses o más.

TABLA II.

\begin{tabular}{|lll|}
\hline & ISD > 24 meses seguimiento. & Recidivas > 24 meses seguimiento. \\
\hline Pacientes & 65 & 95 \\
\hline Seguimiento Meses / Medio & $24-53 / 32.7$ & $24-75 / 33,4$ \\
\hline Curación \# / \% & $54 / 83 \%$ & $82 / 86,3 \%$ \\
\hline Mejoría \# / \% & $7 / 10.7 \%$ & $10 / 10.5 \%$ \\
\hline Fracaso \# / \% & $4 / 6.1 \%$ & $3 / 0.3 \%$ \\
\hline Urgencia De Novo \# / \% & $7 / 10,7 \%$ & $11 / 11,6 \%$ \\
\hline Reajuste Inmediato \# / \% & $45 / 69 \%$ & $48 / 50,5 \%$ \\
\hline Reajuste medio-Largo plazo \# / \% & $7 / 10,7 \%$ & $19 / 20 \%$ \\
\hline Retirada del Varitensor \# / \% & $1 / 1.5 \%$ & $3 / 3,1 \%$ \\
\hline Erosion vaginal/ \# / \% & $1 / 1.5 \%$ & $1 / 0,1 \%$ \\
\hline
\end{tabular}




\section{DISCUSIÓN}

El Sistema Remeex es un dispositivo fabricado en España y aprobado por la FDA para el tratamiento de la incontinencia urinaria de esfuerzo. En este amplio grupo de pacientes no homogéneos podemos ver que el sistema Remeex obtiene buenos resultados y pocas complicaciones, como también se puede observar en otros estudios con grupos homogéneos. $[4,5,6]$.

En este registro participaron Servicios de Urología y Ginecología, así como Hospitales Universitarios y Hospitales Comarcales con un amplio espectro de pacientes como podemos ver en las tablas. Se considera que este artículo es resultado del trabajo en equipo del grupo español Remeex y que no hubiera podido llevarse a cabo sin la participación de todos y cada uno de los autores reflejados.

La técnica del Sistema Remeex (TRT) requiere algo mas de tiempo que las Mallas libres de Tensión, especialmente en el reajuste a la mañana siguiente de la intervención, pero los resultados obtenidos en este registro demuestran que el porcentaje de éxito es elevado, incluso en pacientes recurrentes y con déficit esfinteriano.[7,8].

Podemos observar que el número de pacientes con disfunciones de vaciado fue mínima, solo en tres pacientes se tuvo que disminuir la tensión. Esto fue posible girando el manipulador en sentido antihorario y ayudando el descenso de la uretra mediante un cateter rigido.

Se comprobó que la posibilidad de reajustar funcionó en todos los casos. El Reajuste a medio-largo plazo de pacientes con un seguimiento $>24$ meses fue de un $16.2 \%$, en pacientes con un seguimiento $>42$ meses fue de un $12.9 \%$. Por lo que podemos afirmar que el dispositivo permite ser reajustado a largo plazo adaptando el nivel de soporte uretral del cabestrillo.[9]

La posibilidad de reajustar es un concepto que ha ido ganando aceptación por sus buenos resultados en los tratamientos de incontinencia urinaria. El sistema Remeex es el único que posee publicaciones a largo plazo.[9]

\section{CONCLUSIÓN}

El sistema Remeex es un tratamiento efectivo para la incontinencia urinaria de esfuerzo, aunque requiere de un entrenamiento previo especialmente en el Reajuste. Esta técnica puede utilizarse de forma combinada con otros tipos de cirugía reconstructiva del suelo vaginal. El sistema Remeex es un tratamiento con buenos resultados a largo plazo y podemos considerarlo tratamiento de elección, especialmente en pacientes reintervenidos, pacientes con un déficit intrínseco del esfínter y pacientes con problemas de vaciado. Estudios multicentricos randomizados aportarán mas información al comparar las tasas de éxito y complicaciones, con las mallas libres de tensión.

\section{BIBLIOGRAFÍA Y LECTURAS RECOMENDADAS (*lectura de interés $y^{* *}$ lectura fundamental)}

*1. KHULLAR, V.; SALVATORE, S.; CARDOZO, L.D. y cols.: "The importance of urinary symptoms and urodynamic parameters in quality of life assessment". Neurourol. Urodyn., 14: 540, 1995.

*2. VERSI, E.; CARDOZO, L.; ANAND, D. y cols.: "Symptoms analysis for the diagnosis of genuine stress incontinence". Br. J. Obstet. Gynaecol., 98: $815,1991$.

**3. IGLESIAS, X.; ESPUÑA, M.: "Surgical treatment of urinary stress incontinence using a method for postoperative adjustment of sling tension (Remeex System)". International Urogynecology Journal, Nov., 2003.

**4. CEREZUELA REQUENA, J.F.; MEDINA RAMOS, N.; MARTIN MARTINEZ, A. y cols.: "Analysis of the results of retropubic colpourethrosuspension using a suburethral readjustable sling (REMEEX)".European Journal of Obstet. Gynecol. Reprod. Biol., 106:179, 2003.

**5. ERRANDO, C.; PRADOS, M.; GAUSSA, L. y cols.: "Fundacio Puigvert treatment of stress urinary incontinence with remeex sling". ICS, 345 , 2003.

**6. SOUSA-ESCANDÓN, A.; LEMA GRILLÉ, J.; RODRÍGUEZ GÓMEZ, J.I. y cols.: "Externally readjustable device to regulate sling tension in stress urinary incontinence: preliminary results". J. Endourol., Vol:17, 7: 515, 2004.

*7. REZAPOUR, M.; ULMSTEN, U.: "Tension-free vaginal tape (TVT) in women with recurrent stress urinary incontinence a long term follow-up." International Urogynecology Journal, 2: 9, 2001.

**8. ULMSTEN, U.; ASMUSSEN, M.; LINDSTRÖM, K.: "Tension-free vaginal tape (TVT) in stress incontinent women with intrinsic sphinter deficiency (ISD)- a long -term follow-up". International Urogynecology Journal, 12: 12, 2001.

*9. CEREZUELA J.F.; LUELMO; MEDINA y cols.: "Remeex system: long term follow up". ICS meeting, 